\title{
The impact of curve severity on obstetric complications and regional anesthesia utilization in pregnant patients with adolescent idiopathic scoliosis: a preliminary analysis
}

\author{
Emily W. Chan, BS, ${ }^{1,2}$ Stephen R. Gannon, CCRP, ${ }^{1}$ Chevis N. Shannon, DrPH, ${ }^{1}$ \\ Jeffrey E. Martus, MD, ${ }^{3}$ Gregory A. Mencio, MD, ${ }^{3}$ and Christopher M. Bonfield, MD ${ }^{1,4}$ \\ ${ }^{1}$ Surgical Outcomes Center for Kids, and Departments of ${ }^{3}$ Orthopaedic Surgery and Rehabilitation and ${ }^{4}$ Neurological Surgery, \\ Monroe Carell Jr. Children's Hospital at Vanderbilt, Nashville; and '2Vanderbilt University School of Medicine, Nashville, \\ Tennessee
}

\begin{abstract}
OBJECTIVE Adolescent idiopathic scoliosis (AIS), the most common type of scoliosis, often presents immediately prior to a woman's childbearing years; however, research investigating the impact of AIS on women's health, particularly pregnancy delivery outcomes, is sparse, with existing literature reporting mixed findings. Similarly limited are studies examining the change in scoliotic curve during or after pregnancy. Therefore, this study aims to determine 1) the impact of scoliotic curvature on obstetric complications (preterm births, induction of labor, and urgent/emergency caesarean section delivery), 2) regional anesthetic decision making and success during delivery for these patients, and 3) the effect of pregnancy on curve progression.
\end{abstract}

METHODS Records of all pregnant patients diagnosed with AIS at the authors' institution who delivered between January 2002 and September 2016 were retrospectively reviewed. Demographic information, pre- and postpartum radiographic Cobb angles, and clinical data for each pregnancy and delivery were recorded and analyzed. The Wilcoxon rank-sum test and the Wilcoxon signed-rank test were used for statistical analyses.

RESULTS Fifty-nine patients (84 deliveries) were included; 14 patients had undergone prior posterior spinal fusion. The median age at AIS diagnosis was 15.2 years, and the median age at delivery was 21.8 years. Overall, the median major Cobb angle prior to the first pregnancy was $25^{\circ}\left(\operatorname{IQR} 15^{\circ}-40^{\circ}\right)$. Most births were by spontaneous vaginal delivery $(\mathrm{n}=$ 45 ; $54 \%$ ); elective caesarean section was performed in 17 deliveries (20\%). Obstetric complications included preterm birth $(n=18 ; 21.4 \%)$, induction of labor $(n=20 ; 23.8 \%)$, and urgent/emergency caesarean section $(n=12 ; 14.0 \%)$; none were associated with severity of scoliosis curve or prior spinal fusion. Attempts at spinal anesthesia were successful $99 \%$ of the time (70/71 deliveries), even among the patients who had undergone prior spinal fusion $(n=13)$. There were only 3 instances of provider refusal to administer spinal anesthesia. In the subset of 11 patients who underwent postpartum scoliosis radiography, there was no statistically significant change in curve magnitude either during or immediately after pregnancy.

CONCLUSIONS The results of this study suggest that there was no effect of the severity of scoliosis on delivery complications or regional anesthetic decision making in pregnant patients with AIS. Moreover, scoliosis was not observed to progress significantly during or immediately after pregnancy. Larger prospective studies are needed to further investigate these outcomes, the findings of which can guide the prenatal education and counseling of pregnant patients with AIS.

https://thejns.org/doi/abs/10.3171/2017.7.FOCUS17321

KEY WORDS scoliosis; adolescent idiopathic; pregnancy; delivery outcomes; obstetric complications

A DOLESCENT idiopathic scoliosis (AIS) is the most common type of spinal deformity in adolescents and young adults, affecting $0.4 \%-5.2 \%$ of individuals 10-17 years old. ${ }^{11}$ Both prevalence and curve severity tend to increase with age, with the greatest rate of scoliosis progression occurring during the rapid growth of puber- ty. Moreover, AIS is more common among females than males, especially with greater curve magnitude. ${ }^{11}$ Given that the typical onset of scoliosis occurs immediately prior to a woman's childbearing years, the impact of AIS on women's health is a particularly relevant topic of study.

The limited existing literature investigating delivery 
outcomes in pregnant patients with AIS has yielded conflicting results, especially with regard to complications such as preterm birth, induction of labor, and urgent or emergency caesarian section. Similarly discordant is the research involving the use of spinal anesthesia in these patients, which, in theory, would be more difficult in patients with severe curves or in those who had undergone spinal fusion. ${ }^{1,6,18}$ Another topic of interest is the potential for scoliosis progression during pregnancy, for which research has also been sparse. Of the existing studies regarding AIS and pregnancy, few have taken place in North America, especially over the past 2 decades. As such, this study aims to investigate several of these unanswered questions on topics including 1) the impact of scoliosis on obstetric complications in pregnant patients with AIS (preterm births, the need for labor induction, and caesarean section delivery), 2) regional anesthetic decision making and success during delivery, and 3) the effect of pregnancy on curve progression.

\section{Methods}

After obtaining IRB approval, we reviewed the records of all pregnant patients who had been diagnosed with AIS at our institution, the Monroe Carell Jr. Children's Hospital at Vanderbilt, and who had delivered between January 2002 and September 2016. We identified these patients based on ICD-9 and ICD-10 codes for scoliosis, as well as procedural codes for delivery. Pregnant patients who had been diagnosed with idiopathic scoliosis between 10 and 18 years of age (with or without scoliosis correction surgery), prior to their first pregnancy, were included. Patients who delivered at other institutions, with adequate documentation of the delivery details, were also included. We excluded patients diagnosed with other types of scoliosis (e.g., congenital, neuromuscular), those who had early termination of pregnancy, and mothers who died upon birth.

A REDCap database was created to store and collect data for each pregnancy. Patients' charts were reviewed to obtain demographic information; clinical and radiographic scoliosis data, including pre- and postpartum Cobb angles; and details about spinal fusion, if performed. Cobb measurements were obtained from the treating spine surgeon as documented in the patient's medical record. If this was not available, measurements were performed by the senior author. As intra- and interobserver variability in curve measurements can range from $3^{\circ}$ to $10^{\circ}$, a clinically significant curve change after pregnancy was defined as $\geq 10^{\circ} .4,10,13,14$ Pregnancy and delivery details, including incidence of delivery complications (need for induction or emergency caesarean section), type of anesthesia used during delivery, and pregnancy complications (e.g., preeclampsia) that may have affected the timing or method of delivery were also collected and analyzed.

\section{Statistical Analysis}

Statistical software package $\mathrm{R}$ was used for our data analyses. Descriptive statistics for our patient cohort were calculated. Our data did not fall under a normal distribution; thus, we used the Wilcoxon rank-sum test to determine the association between degree of curvature and
TABLE 1. Patient characteristics

\begin{tabular}{lc}
\hline \multicolumn{1}{c}{ Characteristic } & Value \\
\hline Mean age at scoliosis diagnosis \pm SD, yrs & $14.4 \pm 2.1$ \\
\hline Previous posterior spinal fusion & $14(23.7)$ \\
\hline Median spine curvature prior to pregnancy $($ IQR) & \\
\hline Overall & $25^{\circ}\left(15^{\circ}-40^{\circ}\right)$ \\
\hline No fusion & $23^{\circ}\left(14^{\circ}-38^{\circ}\right)$ \\
\hline Prior fusion & $31^{\circ}\left(19.8^{\circ}-45.5^{\circ}\right)$ \\
\hline Major curve laterality & \\
\hline Right & $44(74.6)$ \\
\hline Left & $14(23.7)$ \\
\hline Major curve level & $48(81.4)$ \\
\hline Thoracic & $10(16.9)$ \\
\hline Lumbar & $38(64.4)$ \\
\hline No. of documented deliveries & $17(28.8)$ \\
\hline 1 & $4(6.8)$ \\
\hline 2 &
\end{tabular}

Values are presented as the number of patients (\%) unless stated otherwise.

incidence of delivery complications, and the Wilcoxon signed-rank test to determine the effect of pregnancy on curve change. Criterion for statistical significance was set at $\mathrm{p}<0.05$.

\section{Results}

\section{Patient Characteristics}

Fifty-one patients met our inclusion criteria, with a total of 84 deliveries. The cohort was predominantly Caucasian $(69.5 \%)$, with a mean age at scoliosis diagnosis of $14.4 \pm 2.1$ years. Overall, the median major Cobb angle prior to the first pregnancy was $25^{\circ}$ (interquartile range [IQR $] 15^{\circ}-40^{\circ}$ ), and 14 patients had previously undergone posterior spinal fusion for correction of their scoliosis. The median major Cobb angle for patients without prior fusion was $23^{\circ}\left(\mathrm{IQR} 14^{\circ}-38^{\circ}\right.$ ) and $31^{\circ}$ (IQR $19.8^{\circ}-45.5^{\circ}$ ) for those with fusion. The majority of patients had a right thoracic major curve (Table 1).

\section{Delivery Outcomes}

Delivery details are illustrated in Table 2. Table 3 shows the comparison of median curve Cobb angle between those with and those without obstetric complications, including preterm delivery, induction of labor, and unplanned caesarean section. There were 18 (21.4\%) preterm births, defined as delivery at less than 37 weeks' estimated gestational age. This was not significantly associated with curve severity $(\mathrm{p}=0.641)$. The majority of the deliveries $(65.5 \%)$ were by vaginal birth, consisting of spontaneous vaginal delivery (54\%), forceps-assisted vaginal delivery $(8 \%)$, and vacuum-assisted vaginal delivery (4\%). Induction was required for 20 deliveries $(23.8 \%)$, which also was not significantly affected by degree of curvature $(\mathrm{p}$ $=0.904)$. The most common reasons for induction were postterm pregnancy and oligohydramnios (Fig. 1). A total 
TABLE 2. Delivery details

\begin{tabular}{cc}
\hline Type of delivery & No. of Patients (\%) \\
\hline Vaginal delivery & $55(65.5)$ \\
\hline Spontaneous & $45(54)$ \\
\hline Forceps-assisted & $7(8)$ \\
\hline Vacuum-assisted & $3(4)$ \\
\hline Caesarean-section delivery & $29(34.5)$ \\
\hline Planned & $17(20)$ \\
\hline Urgent/emergency & $12(14)$ \\
\hline
\end{tabular}

of $29(34.5 \%)$ caesarean sections were performed in our cohort; 12 of these (14\%) were performed on an urgent or emergency basis. The incidence of urgent/emergency caesarean section was not significantly associated with curve severity $(\mathrm{p}=0.181)$. The most common reason for unplanned caesarean section was failure to progress, followed by preeclampsia (Fig. 2).

\section{Spinal Anesthesia}

As shown in Table 4, spinal anesthesia (defined as epidural or spinal block) was administered successfully in $99 \%$ of the deliveries in which it was attempted (70 of 71). Of the remaining cases $(n=13)$, the reasons for lack of spinal anesthesia were patient choice $(n=3)$, anesthesiologist refusal $(n=3)$, rapid labor progression $(n=3)$, emergency caesarean section $(n=3)$, and unknown $(n=1)$. The 3 incidents of anesthesiologist refusal occurred in 2 patients, one of whom had undergone previous spinal fusion. All 3 of these deliveries were by caesarean section.

\section{Postpartum Curve Change}

Postpartum curve measurements were available for 11 patients (17 births), 3 of whom had prior spinal fusion. All postpartum curve measurements had been performed after the patient's last delivery, a median of 3.48 years (IQR 1.58-3.95 years) after the estimated onset of first pregnancy. Most patients (7/11) only had 1 delivery between curve measurements, while 2 patients had 2 deliveries, and another 2 patients had 3 deliveries between curve measurements. Within this subset of 11 patients, the median pre- and postpartum Cobb angles were $39^{\circ}$ (IQR

TABLE 3. Scoliotic curve measurements for patients with and without obstetric complications

\begin{tabular}{lccc}
\hline \multirow{2}{*}{ Complication Type } & \multicolumn{2}{c}{ Median Cobb Angle (IQR), } & $p$ \\
\cline { 2 - 3 } & Complication* $^{\circ}$ & No Complication† & Value \\
\hline Preterm delivery & $28.0(18.0-40.0)$ & $25.0(14.5-40.0)$ & 0.641 \\
\hline Induction of labor & $23.5(14.5-46.2)$ & $25.5(16.0-40.0)$ & 0.904 \\
\hline $\begin{array}{l}\text { Unplanned caesarean } \\
\text { section }\end{array}$ & $18.0(15.0-29.5)$ & $27.0(15.8-42.2)$ & 0.181 \\
\hline
\end{tabular}

* Eighteen patients had preterm delivery, 20 were induced, and 12 had an unplanned caesarean section.

† Sixty-six patients had preterm delivery, 64 were induced, and 72 had an unplanned caesarean section.

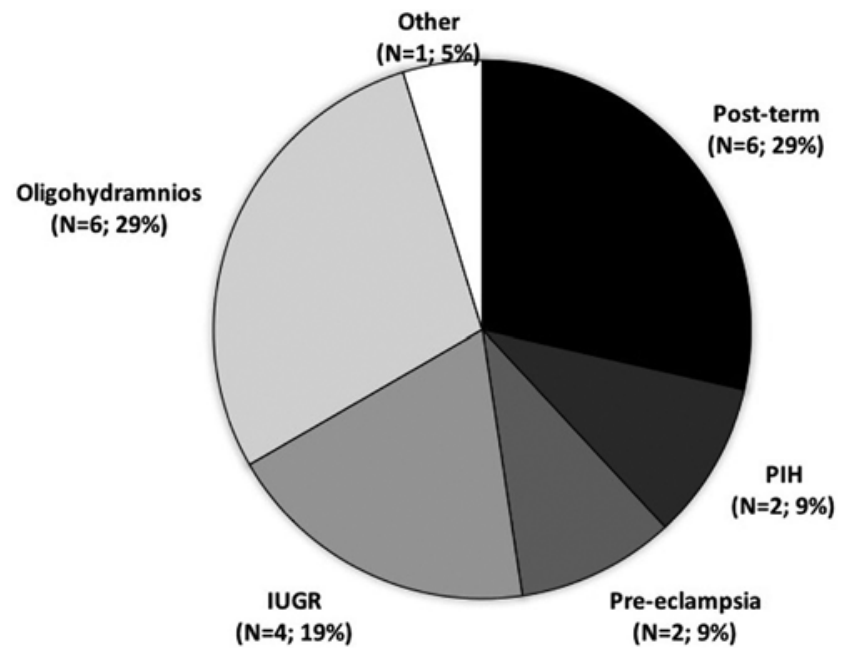

FIG. 1. Indications for induction $(n=20)$. Note that some patients may have required induction for more than 1 condition. IUGR = intrauterine growth restriction; $\mathrm{PIH}=$ pregnancy-induced hypertension; postterm = more than 40 weeks gestation.

$29^{\circ}-58^{\circ}$ ) and $40^{\circ}\left(\right.$ IQR $28^{\circ}-60^{\circ}$ ), respectively. No significant change was demonstrated $(\mathrm{p}=0.247)$. On individual case assessment, the vast majority of patients (10/11) did not have a clinically significant curve change. However, 1 patient had a $10^{\circ}$ curve increase after a single delivery (from $30^{\circ}$ to $40^{\circ}$ ).

\section{Discussion}

In summary, this retrospective study of pregnant patients with preexisting AIS investigated the effects of scoliotic curvature on delivery outcomes, as well as the impact of pregnancy on change in spine curvature. The results suggest that there was no effect of the severity of

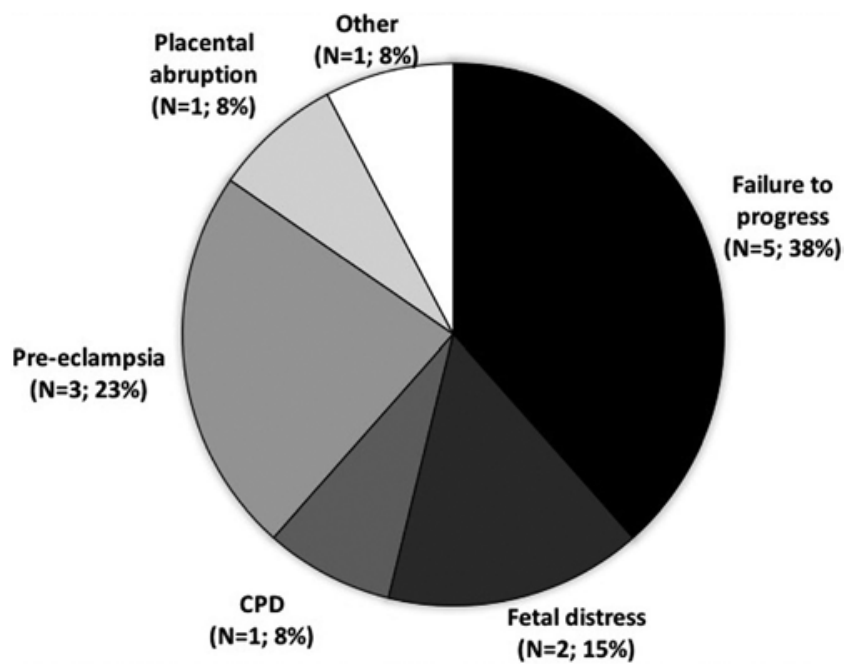

FIG. 2. Indications for urgent/emergency caesarean section $(n=12)$. Note that some patients may have required urgent/emergency caesarean section for more than 1 condition. CPD = cephalopelvic disproportion. 
TABLE 4. Spinal anesthesia outcomes

\begin{tabular}{cc}
\hline Spinal Anesthesia Attempted & No. of Patients (\%) \\
\hline Yes & $71(84.5)$ \\
\hline Successful & $70(99)^{*}$ \\
\hline Not successful & $1(1)^{\star}$ \\
\hline No & $13(15.5)$ \\
\hline Patient choice & $3(3.6)$ \\
\hline Anesthesiologist refusal & $3(3.6) \dagger$ \\
\hline Rapid labor progression & $3(3.6)$ \\
\hline Emergency caesarean section & $3(3.6)$ \\
\hline Unknown & $1(1.1)$ \\
\hline
\end{tabular}

* Percentages are based on the number of attempts.

† Occurred in 2 patients, 1 of whom had undergone previous spinal fusion.

scoliosis on delivery complications or regional anesthetic decision making in pregnant patients with AIS. The incidence of preterm birth is slightly higher than the national average; however, it was not associated with severity of scoliosis. Overall, the caesarean section rate was similar to that of the national average. In only 1 patient in whom spinal anesthesia was attempted was the procedure not successful. Moreover, scoliosis was not observed to progress significantly during or immediately after pregnancy.

The incidence of preterm birth in our study was $21.4 \%$, which is higher than the national preterm birth rate $(9.6 \%$ $10.4 \%$ during the time of study); $;$ however, preterm birth was not associated with severity of scoliosis. The increased rate of preterm deliveries may be due to a greater number of patients with high-risk pregnancies seeking care at a specialized, tertiary care facility such as Vanderbilt. The rate of labor induction was $23.8 \%$ in our population, which is similar to that of the national average $(20.9 \%-23.8 \%) .{ }^{16}$ It was also not associated with scoliotic curve severity, suggesting that patients with more severe scoliosis were not more likely to require induction. Our results are supported by Lebel et al., who found that after accounting for nulliparity, fertility treatment, and maternal age, labor induction was not associated with scoliosis. ${ }^{12}$ Our cohort had an overall caesarean section rate of $34.5 \%$, which is similar to the national average (26.1\%-32.9\%). ${ }^{17}$ The incidence of urgent/emergency caesarean section was $14 \%$. We expected that this complication would be more likely in patients with severe scoliosis, as the altered anatomy of the spine might cause arrest of fetal descent. Interestingly, there was not an association between degree of curvature and urgent/emergency caesarean section. Although previous studies have not specifically examined unplanned caesarean sections, several studies have reported that the overall rate of caesarean section is not increased in patients with AIS compared with age-matched controls. ${ }^{5,7}$ Overall, our study found that the severity of scoliosis did not increase the risk for preterm birth, need for induction, or urgent/emergency caesarean section. Further study is needed in this area, especially with respect to the incidence of unplanned caesarean section in this population.

Anesthetic decision making for delivery is another area of uncertainty in the AIS literature. Some anesthesiolo- gists might be reluctant to administer spinal anesthesia in patients with severe scoliosis, as the curvature of the spine might cause difficulty in locating anatomical landmarks for epidural placement. Patients who have previously undergone spinal surgery present an even greater challenge; research has shown that these patients have a greater failure rate for placement and longer procedure time than patients without prior spinal surgery. ${ }^{1,6}$ In addition, scarring of the epidural space due to spinal surgery may reduce the efficacy of epidural anesthesia. ${ }^{18}$ In our study, there were only 3 instances of physician refusal to attempt spinal anesthesia, 2 of which were for the same patient who had previously undergone spinal fusion. In only 1 patient was the attempted placement of an epidural unsuccessful. Due to the low number of cases with this outcome, it is difficult to draw definitive conclusions, although it does not appear that anesthetic decision making is affected by degree of curvature or previous spinal surgery. These results are similar to those of Betz et al., who reported that the failure of spinal anesthesia placement due to scoliosis was only observed in 2 of 159 deliveries in patients with idiopathic scoliosis. ${ }^{2}$ Other studies have reported opposing results; for example, Falick-Michaeli et al. found that $70 \%$ of patients who had undergone prior spinal fusion for AIS were refused spinal anesthesia. ${ }^{8}$ It should be noted, however, that this study was limited by its sample size $(\mathrm{n}=17)$, and it was conducted in Israel, where standard anesthetic practices and specialist experience may differ from institutions in the United States. The low rate of anesthesiologist refusal in our study may reflect the technical expertise and comfort level of providers at a large academic institution. Future studies might consider examining larger populations across multiple centers.

The final outcome of interest was the effect of pregnancy on change in the scoliotic curve. This study did not show any overall significant change in Cobb angle for the 11 patients who underwent postpartum radiography. This may reflect complete stability of the prepartum curve in these patients, as Blount and Mellencamp found a similar lack of progression in patients whose curve had been stable (i.e., no increase in severity after conclusion of treatment) before pregnancy. ${ }^{3}$ However, the lack of observable curve change may also be due to our small sample size. Previous research has shown that there may be some increase in scoliotic curve after pregnancy in patients both with and without previous spinal fusion; $5,7,15$ however, these increases were very rarely $\geq 10^{\circ}$, consistent with our findings. Additionally, Betz et al. found that while some patients with AIS had an increase in curve severity after pregnancy, the change was similar to the natural progression of scoliosis in patients who had never been pregnant. ${ }^{2}$ Thus, as it currently stands, evidence from previous research and from our study suggest that pregnancy does not have a detrimental effect on scoliotic curve.

The results of this study should be interpreted with caution, as it has some limitations. First, as with all retrospective studies, there is an inherent risk of incomplete data, particularly with the postpartum radiographs, which were only performed in 11 patients, due to the fact that obtaining routine scoliosis radiographs into adulthood is not standard of care at our institution for patients with a stable 
curve. As such, only the patients with clinically progressive or severe curves would have undergone radiographic follow-up, leading to the potential for selection bias. In addition, the small number of patients with postpartum radiographs might have underpowered our curve change analysis, leading to the lack of a statistically significant change in Cobb angle after pregnancy. Despite these limitations, our study provides important preliminary data and sets the stage for future, prospective research in this area, possibly involving multiple centers. Findings of such studies will be invaluable to spine surgeons and obstetric providers as they counsel patients both during the planning of conception and throughout the course of pregnancy.

\section{Conclusions}

The results of our study suggest that there is no effect of severity of scoliosis curve on delivery complications or anesthetic decision making in pregnant patients with AIS. Moreover, the scoliosis curve size did not significantly increase after the pregnancy. Larger, prospective studies are needed to further investigate these outcomes, the findings of which can guide the prenatal education and counseling of pregnant patients with AIS.

\section{References}

1. Bauchat JR, McCarthy RJ, Koski TR, Wong CA: Labor analgesia consumption and time to neuraxial catheter placement in women with a history of surgical correction for scoliosis: a case-matched study. Anesth Analg 121:981-987, 2015

2. Betz RR, Bunnell WP, Lambrecht-Mulier E, MacEwen GD: Scoliosis and pregnancy. J Bone Joint Surg Am 69:90-96, 1987

3. Blount WP, Mellencamp D: The effect of pregnancy on idiopathic scoliosis. J Bone Joint Surg Am 62:1083-1087, 1980

4. Carman DL, Browne RH, Birch JG: Measurement of scoliosis and kyphosis radiographs. Intraobserver and interobserver variation. J Bone Joint Surg Am 72:328-333, 1990

5. Cochran T, Irstam L, Nachemson A: Long-term anatomic and functional changes in patients with adolescent idiopathic scoliosis treated by Harrington rod fusion. Spine (Phila Pa 1976) 8:576-584, 1983

6. Crosby ET, Halpern SH: Obstetric epidural anaesthesia in patients with Harrington instrumentation. Can J Anaesth 36:693-696, 1989

7. Danielsson AJ, Nachemson AL: Childbearing, curve progression, and sexual function in women 22 years after treatment for adolescent idiopathic scoliosis: a case-control study. Spine (Phila Pa 1976) 26:1449-1456, 2001

8. Falick-Michaeli T, Schroeder JE, Barzilay Y, Luria M, Itzchayek E, Kaplan L: Adolescent idiopathic scoliosis and pregnancy: an unsolved paradigm. Global Spine J 5:179184, 2015 (Erratum in Global Spine J 6:626, 2016)
9. Hamilton BE, Martin HA, Osterman MJK, Curtin SC, Mathews TJ: Births: final data for 2014. Nat Vital Stat Rep 64:1-64, 2015

10. Kim H, Kim HS, Moon ES, Yoon CS, Chung TS, Song HT, et al: Scoliosis imaging: what radiologists should know. Radiographics 30:1823-1842, 2010

11. Konieczny MR, Senyurt H, Krauspe R: Epidemiology of adolescent idiopathic scoliosis. J Child Orthop 7:3-9, 2013

12. Lebel DE, Sergienko R, Wiznitzer A, Velan GJ, Sheiner E: Mode of delivery and other pregnancy outcomes of patients with documented scoliosis. J Matern Fetal Neonatal Med 25:639-641, 2012

13. Loder RT, Spiegel D, Gutknecht S, Kleist K, Ly T, Mehbod A: The assessment of intraobserver and interobserver error in the measurement of noncongenital scoliosis in children < or $=10$ years of age. Spine (Phila Pa 1976) 29:2548-2553, 2004

14. Morrissy RT, Goldsmith GS, Hall EC, Kehl D, Cowie GH: Measurement of the Cobb angle on radiographs of patients who have scoliosis. Evaluation of intrinsic error. J Bone Joint Surg Am 72:320-327, 1990

15. Orvomaa E, Hiilesmaa V, Poussa M, Snellman O, Tallroth $\mathrm{K}$ : Pregnancy and delivery in patients operated by the Harrington method for idiopathic scoliosis. Eur Spine J 6:304307, 1997

16. Osterman MJK, Martin JA: Recent declines in induction of labor by gestational age. NCHS Data Brief 155:1-8, 2014

17. Osterman MJK, Martin JA: Trends in low-risk cesarean delivery in the United States, 1990-2013. Natl Vital Stat Rep 63:1-16, 2014

18. Smith PS, Wilson RC, Robinson AP, Lyons GR: Regional blockade for delivery in women with scoliosis or previous spinal surgery. Int J Obstet Anesth 12:17-22, 2003

\section{Disclosures}

The authors report no conflict of interest concerning the materials or methods used in this study or the findings specified in this paper.

\section{Author Contributions}

Conception and design: all authors. Acquisition of data: Chan, Shannon. Analysis and interpretation of data: Bonfield, Chan, Shannon. Drafting the article: Bonfield, Chan. Critically revising the article: all authors. Reviewed submitted version of manuscript: all authors. Approved the final version of the manuscript on behalf of all authors: Bonfield. Statistical analysis: Chan. Administrative/technical/material support: Bonfield, Gannon, Shannon. Study supervision: Bonfield, Gannon, Shannon.

\section{Correspondence}

Christopher M. Bonfield, Department of Neurological Surgery, Monroe Carell Jr. Children's Hospital at Vanderbilt, 2200 Children's Way, DOT \#9226, Nashville, TN 37232. email: chris. bonfield@vanderbilt.edu. 